is $\mathrm{I}-3,000$; and for para $\mathrm{B} \mathrm{I}-300$. The third series immunized with para $B$ shows its highest lytic dilution with a heterologous strain, typhoid (I-950); while for para A and para B the corresponding dilutions are $\mathrm{I}-380$ and $\mathrm{I}-350$ respectively.

The fourth series of rabbits was immunized with combined triple vaccine, and the degree of immunity reached for each organism (as measured by the highest lytic dilution) would appear to be lower than the degree of immunity obtained for the same organism by inoculation with the single strain vaccine. This result was not wholly anticipated, from the previous work of Castellani' and also of Davison. ${ }^{2}$ The latter in a series of very completely worked out experiments with agglutinins, reports that the combined vaccine when injected, gives for each organism as good and usually a greater immunity response than if it had been injected alone. Davison, and likewise Bull ${ }^{3}$ have also observed some tendency in respect to the heterologous strains to lead to an added formation of the specific agglutinin, a phenomenon somewhat comparable to that of "summation" in muscle contraction. Our results with bacteriolysins, in a small series of animals, if confirmed, would appear to differ from Davison's observations with agglutinins. Further experimentation along these lines is desirable, and it is our hope to amplify our own work with improved methods.

\title{
$102(1562)$
}

\section{Studies on intestinal implantation of Bacillus acidophilus.}

\section{By Harry A. Cheplin and Leo F. Rettger.}

[From Yale University, New Haven, Conn.]

B. acidophilus (Moro) is a common inhabitant of the intestinal tract of the albino rat and of man. Ordinarily it is present in very small numbers, however, and of ten may escape detection.

Diet exerts a profound influence on the character of the intestinal flora. Lactose and dextrin, when fed in sufficient amounts, bring about a marked transformation in bacterial types.

1 Ibid. and Centralbl. Bakt., I909, Ab. I, 52, p. 92.

${ }^{2}$ Davison, W. C., Arch. of Inter. Medicine, I918, XXI, 437.

3 Bull, C. G., Jour. Exp. Med., 1916, XXIII, 419. 
The present investigation has shown, also, that the ingestion of pure cultures or suspensions of $B$. acidophilus results in a similar change.

The administration of 2 grams of either lactose or dextrin to white rats, in connection with a basal diet of bread and meat, caused within three to six days a complete transformation of the fecal flora from the ordinary mixed type to one strongly dominated by $B$. acidophilus, while the same amounts of maltose, sucrose and glucose failed to exert any transforming influence. The ingestion of I gram of lactose or dextrin brought about a partial change, whereas the addition of I c.c. (nephelometer 5) of living cultures of B. acidophilus to the I gram of carbohydrate effected a complete simplification of the intestinal flora. Identical results were obtained by the administration of 2 c.c. of the $B$. acidophilus culture or suspension alone. Post-mortem examinations of the different sections of the alimentary canal of rats harboring a simplified flora of the aciduric type revealed a general distribution of $B$. acidophilus throughout the length of the intestine.

The implantation of $B$. bulgaricus was not effected by the ingestion of even 5 c.c. of $B$. bulgaricus suspension of the same concentration as those of $B$. acidophilus, either with or without accompanying lactose. However, when $B$. bulgaricus and lactose were given simultaneously a transformation of the intestinal flora took place corresponding to that obtained with the feeding of 2 grams of lactose alone.

In feeding experiments with human subjects it has likewise been shown that either lactose or dextrin, when added in sufficient amounts to the ordinary diet, causes a pronounced transformation of the intestinal flora within four to six days, with a marked predominance of $B$. acidophilus. With a single exception thus far, the ingestion of 300 grams of either of these carbohydrates led to such a proliferation of $B$. acidophilus that it entirely dominated the flora and effected an almost complete suppression of all other viable bacterial types. The simple character of the flora tended to remain permanent so long as the ingestion of these carbohydrates was continued. The administration of 400 grams was necessary in the one exception noted. 
No radical suppression of the commonly prevailing types was observed when but 150 grams of lactose or dextrin were ingested, barring two notable exceptions. The addition of 150 c.c. of living cultures of $B$. acidophilus to the 150 grams of lactose or dextrin caused a very pronounced transformation of the fecal flora from the usual mixed type to one strongly dominated by $B$. acidophilus. The daily ingestion of 300 c.c. of $B$. acidophilus suspension alone effected simplification of the flora, reducing it to the aciduric type.

The striking results obtained from the use of milk soured with $B$. acidophilus bring to view a new avenue of approach to the field of $B$. acidophilus implantation within the alimentary canal. The administration per os of one liter of $B$. acidophilus milk, in conjunction with the ordinary daily diet, exercised in every instance a telling effect upon the complex flora, which was clearly manifested within one to six (usually less than three) days in the establishment of a non-gas-producing intestinal flora dominated by B. acidophillis. On the other hand, the ingestion of a similar amount of $B$. bulgaricus milk offered no encouragement whatever to the aciduric type of organisms. In a few instances the consumption of only 500 c.c. of $B$. acidophilus milk was sufficient to establish a $B$. acidophilus flora. The addition of Ioo grams of lactose or dextrin to 500 c.c. of $B$. acidophilus milk resulted in a rapid simplification of types in those subjects who did not respond readily to the ingestion of the 500 c.c. of milk culture alone.

There appears to be a definite correlation between the rate of absorption in the alimentary canal of a utilizable carbohydrate and its tendency to effect a transformation of the intestinal flora. This relation was indicated in the observation that the feces of lactose- and dextrin-fed rats contained reducing substances at the times when $B$. acidophilus was present in preponderating numbers, while the feces of the animals receiving maltose, sucrose or glucose gave negative results with Benedict's solution and presented no change in the types of bacteria. With the human subjects results were obtained after lactose ingestion similar to those furnished by the albino rats.

No definite relation could be established, on the other hand, 
between the hydrogen ion concentration of the feces and the bacterial flora. That is to say, the hydrogen ion concentration limits remained essentially the same during the preliminary and the transforming periods of the different experiments.

\section{$103(1563)$}

An unrecognized pathway for bacterial invasion of the respiratory tract.

By M. C. Winternitz, G. H. Smith and E. S. Robinson (by invitation).

[From the Brady Laboratory of Pathology and Bacteriology, Yale University School of Medicine, Nerw Haven, Conn.]

Normally, the ciliated, mucus-secreting epithelium is a mechanism competent to protect the lungs against infection by way of the upper respiratory tract. When this epithelium is injured by toxic gas or when the mechanism is otherwise incapacitated, as for example, in aspiration pneumonia, the lumen of the trachea undoubtedly is the pathway traveled by the agent responsible for the pulmonary inflammation. Pneumonia may occur, however, and this is especially true of the lobar type, without demonstrable gross lesion of the upper respiratory tract, and in these circumstances some route other than the above must have been provided.

Experimentally, the introduction of pneumococci by intratracheal instillation or by needle puncture of the tracheal wall through the neck, may result in pneumonia. With either of these methods of inoculation, local damage to the mucosa of the trachea occurs. When the needle method is employed, an opportunity is at hand, not only for infection of the submucosa, but of the peritracheal tissue as well. When the organisms are introduced by insufflation into the rabbit, damage to the mucosa of the larynx or upper trachea can hardly be avoided. In either case, an atrium of invasion for the submucosa of the trachea is provided, and histologically infection of the submucosa is evident at the point of inoculation. From here it may be traced throughout the submucosa of the trachea and larger bronchi to the hilum of the 